\title{
Synchronous Carcinoma of the Ampulla of Vater and Colon Cancer
}

\author{
Anastasios J. Karayiannakis ${ }^{a}$ Stylianos Kakolyris ${ }^{b}$ \\ Georgios Kouklakis ${ }^{c}$ Nikolaos Xenidis ${ }^{b}$ Helen Bolanaki ${ }^{a}$ \\ Christos Tsalikidis $^{\mathrm{a}}$ Constantinos Simopoulos $^{\mathrm{a}}$ \\ aSecond Department of Surgery, bepartment of Clinical Oncology and \\ 'Gastrointestinal Endoscopy Unit, Democritus University of Thrace, Medical \\ School, Alexandroupolis, Greece
}

\section{Key Words}

Ampullary carcinoma - Colorectal carcinoma - Papilla of Vater carcinoma · Second primary cancer Synchronous cancer

\begin{abstract}
Carcinoma of the papilla of Vater is a relatively rare tumor and its coexistence with other primary sporadic cancers is very exceptional. Here we report the case of a 76-year-old man who presented with painless obstructive jaundice, pathologically elevated liver function tests and increased serum levels of carbohydrate antigen 19-9 and carcinoembryonic antigen. Endoscopic retrograde cholangiography revealed a large polypoid mass in the ampulla of Vater. A large tumor in the ascending colon was also incidentally detected by abdominal computed tomography. Endoscopic biopsies from both lesions showed adenocarcinomas. Metastases to the liver and to the hepatoduodenal ligament and hepatic artery lymph nodes were found during surgery. Right colectomy and a biliary bypass were performed. Histological analysis showed an ampullary adenocarcinoma with metastases to regional lymph nodes and the liver and a colonic adenocarcinoma with local invasion into the pericolic fat. Treatment with gemcitabine plus cisplatin was suggested postoperatively. The association of sporadic ampullary and colonic adenocarcinomas and the mutually increased risk of developing either a synchronous or a metachronous tumor following each other should be considered in patients with primary ampullary or colorectal cancer during the preoperative evaluation and postoperative follow-up of these patients.
\end{abstract}

Anastasios J. Karayiannakis, $\mathrm{MD}, \mathrm{MSc}, \mathrm{PhD}$
Second Department of Surgery, Democritus University of Thrace, Medical Schoo GR-68 100 Alexandroupolis (Greece)

Tel. +30 25510 74006, E-Mail akarayan@usa.net 


\section{Introduction}

Carcinoma of the papilla of Vater is a relatively uncommon tumor accounting for about $1 \%$ of all adenocarcinomas and approximately for $5 \%$ of gastrointestinal tract carcinomas. These tumors are diagnosed early because they produce clinical signs or symptoms of biliary obstruction at an early stage due to their location within the biliary tract, and have a good prognosis after surgical resection compared to other periampullary carcinomas, namely bile duct and pancreatic cancers. Ampullary carcinomas can be either sporadic or may be part of several inherited cancer syndromes where they may associate with colorectal cancer. Hereditary nonpolyposis colorectal cancer syndrome and familial adenomatous polyposis are two autosomal dominant syndromes of high penetrance, where extracolonic tumors such as endometrial, ovarian, gastric, intestinal, pancreatic and biliary tract cancers associate with colorectal cancer [1-4]. Peutz-Jeghers syndrome is also an autosomal dominant disorder characterized by the development of hamartomatous polyps in the entire gastrointestinal tract and mucocutaneous lesions of the lips and buccal mucosa, and associated with increased risk of both extraintestinal and gastrointestinal cancers most commonly in the colon and the duodenum. However, there is evidence that sporadic ampullary carcinomas may also associate either synchronously or metachronously with other malignancies such as sporadic colorectal carcinomas $[5,6]$. In a population-based study, patients with ampullary cancer were found to be at increased risk for a second primary colorectal cancer and patients with colorectal cancer were found to be at increased risk for a second primary ampullary carcinoma [7]. This bidirectional, greater than chance, mutual association between these two cancers suggests common genetic, environmental or predisposing risk factors or shared mechanisms of carcinogenesis [7]. Here we describe a rare case of synchronous association of sporadic carcinoma of the papilla of Vater with sporadic colon cancer.

\section{Case Report}

A 76-year-old man was admitted because of painless obstructive jaundice of one week duration when he noticed yellow-colored scleras, dark-colored urine and stool discoloration. He also reported general fatigue, loss of appetite and weight loss $(10 \mathrm{~kg})$ over the last three months. There were no changes in his bowel movements and his past medical history and family history were negative for malignancy or familial cancer syndrome. On physical examination the skin and sclera appeared yellow. Abdominal examination revealed mild tenderness in the right upper abdominal quadrant, but no mass was palpable. There were no enlarged neck, axillary or inguinal lymph nodes.

Laboratory tests revealed microcytic, hypochromic anemia, hematocrit $32.6 \%$ (reference range 40-54\%), hemoglobin $11.1 \mathrm{~g} / \mathrm{dl}$ (reference range 13.5-17.5 g/dl), mean corpuscular volume $74.2 \mathrm{fl}$ (reference range 76-96 fl), mean corpuscular hemoglobin $25.4 \mathrm{pg}$ (reference range 27-34 pg), mean corpuscular hemoglobin concentration $31.3 \mathrm{~g} / \mathrm{dl}$ (reference range $25-32 \mathrm{~g} / \mathrm{dl}$ ) and serum ferum and ferritin levels of $23 \mu \mathrm{g} / \mathrm{dl}$ (reference range $70-180 \mu \mathrm{g} / \mathrm{dl}$ ) and $80 \mu \mathrm{g} / \mathrm{l}$ (reference range 30-400 $\mu \mathrm{g} / \mathrm{l}$ ), respectively. Liver function tests were all abnormally elevated: total bilirubin $13.6 \mathrm{mg} / \mathrm{dl}$, direct bilirubin $10.7 \mathrm{mg} / \mathrm{dl}$, alkaline phosphatase 1,066 U/l, aspartate aminotransferase $201 \mathrm{U} / \mathrm{l}$, alanine aminotransferase $329 \mathrm{U} / \mathrm{l}$, and $\gamma$-glutamyl transpeptidase $470 \mathrm{U} / \mathrm{l}$. Serum levels of carbohydrate antigen $19-9$ and carcinoembryonic antigen were elevated at $540 \mathrm{U} / \mathrm{ml}$ (reference range $0-37 \mathrm{U} / \mathrm{ml}$ ) and $9.2 \mathrm{ng} / \mathrm{ml}$ (reference range $0.9-5.4 \mathrm{ng} / \mathrm{ml}$ ), respectively. All other laboratory parameters were within normal limits and hepatitis B and C serology tests were negative.

Abdominal ultrasonography showed a distended gallbladder, dilatation of the intra- and extrahepatic bile ducts but not gallstones. The performed computed tomography of the abdomen showed enlargement of the pancreatic head suggesting the presence of a tumor either in the pancreatic head or in the periampullary region (fig. 1). An irregular, 6-cm mass in the ascending colon with partial 
luminal obstruction was also incidentally found (fig. 2). There were neither metastatic lesions in the liver nor enlarged intraabdominal lymph nodes or ascites. An endoscopic retrograde cholangiography revealed a large polypoid mass in the ampulla of Vater (fig. 3). Endoscopic biopsies were taken and showed a poorly differentiated adenocarcinoma. Total colonoscopy showed no polyps but a large, ulcerated polypoid mass was found in the ascending colon. Biopsies were taken and the histology showed an adenocarcinoma.

At operation, a large tumor in the ascending colon was found. In addition, five small-sized $(2-3 \mathrm{~mm})$ metastatic lesions were seen in the hepatic surface. Enlarged lymph nodes were present at the hepatic hilus, along the hepatoduodenal ligament and common hepatic artery. Intraoperative frozen sections from the hepatic lesions and the enlarged lymph nodes were both positive for adenocarcinoma. A decision was made to perform right colectomy because of anemia due to chronic blood loss and the possibility of bowel obstruction because of the large size of the tumor. A side to side hepaticojejunostomy was also performed for jaundice relief. Final histological analysis showed a moderately differentiated, mucinous adenocarcinoma of the colon with local invasion into the pericolic fat but without regional lymph node metastases (T3N0M0). The histological features of the examined lymph nodes and hepatic specimens were consistent with those of the ampulla of Vater lesion. Based on these findings, the diagnosis of adenocarcinoma of the ampulla of Vater with metastases to regional lymph nodes and the liver was made and the tumor was classified as stage IV (T3N1M1) according to the sixth edition (2002) of the TNM system. The patient's postoperative course was uneventful and he was discharged on the eighth postoperative day. Treatment with gemcitabine plus cisplatin was proposed, but the patient refused any further treatment and eventually died two months later.

\section{Discussion}

Patients with malignant neoplasms are more likely to develop a second malignancy compared to the general population because of genetic susceptibility, due to common etiologic factors or as a result of treatment for the first cancer. Tumors of the papilla of Vater may be part of several inherited syndromes of polyposis or nonpolyposis colon cancer [1-4]. However, there is evidence that ampullary carcinoma is associated with a high incidence of other malignancies such as colorectal carcinomas [5-7] although case series failed to detect an association between ampullary and colorectal cancer $[8,9]$.

Data from the Surveillance, Epidemiology, and End Results (SEER) Program showed a significantly elevated risk of colorectal cancer in women (relative risk (RR): 2.25, 95\% confidence interval (CI): 1.16-3.94) but not in men (RR: 1.28, 95\% CI: 0.58-2.42) diagnosed with bile duct cancer [6]. Several large population-based studies evaluated the association between noncolorectal and colorectal cancer in the reverse direction, namely the risk of a subsequent primary cancer following colorectal cancer. Data analysis from the SEER Program for the period 1975 through 2001 showed a significantly increased risk for both synchronous and metachronous occurrence for intestinal, gastric, kidney, prostate, female breast, and corpus uteri cancers [10]. Regarding hepatobiliary cancers, there was no overall increased risk for metachronous pancreatic or gallbladder cancer. However, significantly elevated risk for pancreatic cancer was found in younger (less than 50 years) patients. Interestingly, there were no cases of either synchronous or metachronous ampullary carcinomas in this study [10] or in colorectal cancer patients from the Thames Cancer Registry database [11] or from the Swedish Family-Cancer database [12]. However, these studies were not designed to evaluate the association between ampullary and colorectal cancers. Differences among cancer registries regarding cohort characteristics, disease reporting, synchronous or metachronous definition, length of follow-up and coding policy may also account for these discrepancies. Nevertheless, an excess risk of secondary ampullary cancer (standardized incidence ratio (SIR): 3.43; 95\% 
CI: 1.37-7.06) following colon cancer was reported among five-year survivors [8]. Analysis of colorectal cancers from the SEER Program for the 1973-1993 period revealed a significantly increased risk of bile duct cancer in both men (RR: 1.96, 95\% CI: 1.42-2.63) and women (RR: 1.59, 95\% CI: 1.06-2.30) following colorectal cancer [6]. When data from the 1973-1999 period of the SEER Program were analyzed to specifically examine the association between ampullary and colorectal cancer, it was found that among 2,043 white patients with ampullary cancer, a total of 30 developed colorectal cancer over 5,674 person-years of follow-up, as compared with an expected number of 14 patients, thus yielding an overall SIR of 2.14 with a 95\% CI of 1.45-3.06 [7]. The tumors were equally distributed along the colon and rectum in a pattern similar to that observed in the overall colorectal cancer SEER population. Eleven patients had localized disease, 9 patients had regional and 5 patients had metastatic disease. The median time from ampullary cancer diagnosis until diagnosis of the colorectal cancer was 5.25 years (25th-75th percentile: 2.42-10.25 years) with 9 cases diagnosed within one year after diagnosis of the initial ampullary carcinoma. In contrast, 57 out of 262,066 white patients with colorectal cancer developed ampullary cancer as a second primary malignancy with an expected number of 26 ampullary malignancies in this cohort (SIR: 2.18, 95\% CI: 1.69-2.85). The median time between colorectal and subsequent ampullary cancer diagnosis was 7.58 years (25th-75th percentile: $2.33-13.3$ years) with 10 ampullary cancers detected within one year after diagnosis of the initial primary colorectal cancer.

In our patient, a mucinous carcinoma of the colon and a poorly differentiated metastatic adenocarcinoma of the ampulla of Vater were diagnosed simultaneously. We considered these two cancers to be synchronous because they were histologically distinct without evidence of one being a metastasis of the other. They also appeared to be sporadic because a familial cancer syndrome was excluded by history and gastrointestinal polyposis was not found by endoscopy. SEER population-based studies showed a bidirectional association between ampullary and colorectal cancer for both genders with ampullary cancer patients having a risk for the development of colorectal cancer as a second primary malignancy of 2 and vice versa (SIR: 2.14 and 2.18, respectively). Interestingly, the second tumor was diagnosed within one year after diagnosis of the initial primary in $30 \%$ ( 9 out of 30 patients) of ampullary cancer patients and in 17.5\% (10 out of 57 patients) of colorectal cancer patients, suggesting coincidently present tumors [7]. Synchronously diagnosed cancers may be the result of 'lead time bias' since the second tumor may be detected while asymptomatic because of increased medical scrutiny. In addition, when two cancers are diagnosed at the same time, which is designated as the index tumor might be arbitrarily defined as in our patient who presented with symptoms from the ampullary cancer whereas the colon cancer was diagnosed incidentally during the diagnostic work-up. Nevertheless, the association of a frequent cancer like colon cancer with ampullary cancer which is less frequent, the localized feature of the colon cancer as opposed to the metastatic ampullary cancer, along with morphological data from histological analysis and the course of the disease in this patient imply ampullary carcinoma as being the primary tumor. The bidirectional association between ampullary and colorectal cancer with almost equal SIRs suggests similar or common risk factors and mechanisms of carcinogenesis in these two tumors. Sporadic ampullary carcinomas are considered to arise from a preexisting adenoma similarly to the adenoma-carcinoma sequence in colorectal cancer [13-15]. They show histological features of a tubular colorectal adenocarcinoma and are characterized by a $K$-ras mutational rate and molecular alterations similar to that observed in colon cancer $[16,17]$. This similarity 
of biological characteristics with colonic carcinomas may explain at least in part the growth pattern of ampullary carcinomas and the more favorable outcome as opposed to pancreatic and biliary tract carcinomas. When two primary cancers are diagnosed simultaneously, the systemic treatment should target the tumor with worst prognosis. In our patient systemic chemotherapy for metastatic ampullary cancer consisting of cisplatin plus gemcitabine combination was suggested because of the more advanced disease compared to the colon cancer.

In summary, we report a synchronous detection of both presumably sporadic ampullary and colonic adenocarcinomas. The association of carcinoma of the papilla of Vater with colorectal cancer and the mutually increased risks of developing either a synchronous or metachronous tumor following each other should be considered in patients with primary ampullary or colorectal cancer during the preoperative evaluation and postoperative follow-up of these patients.

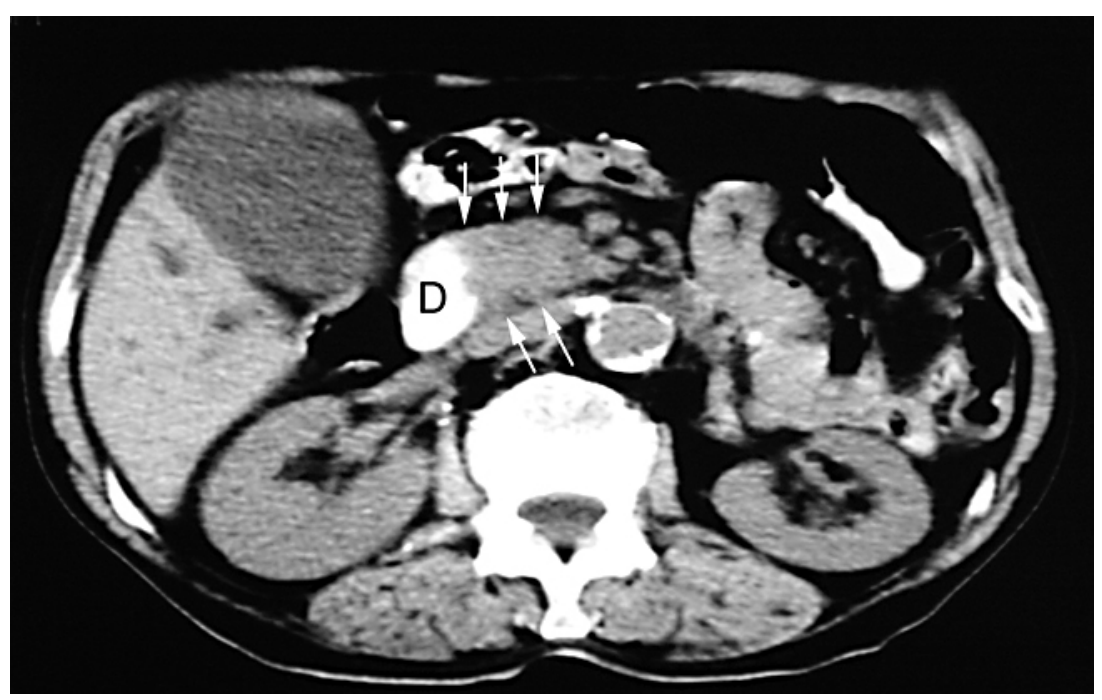

Fig. 1. Abdominal computed tomography scan showing enlargement of the pancreatic head (arrows) indicative of a tumor either in the pancreatic head or in the periampullary region. $\mathrm{D}=$ Duodenum. 


\begin{tabular}{|c|c|c|c|}
\hline $\begin{array}{l}\text { Case Reports in } \\
\text { Gastroenterology }\end{array}$ & \begin{tabular}{|l} 
Case Rep Gastroenterol 2011;5:301-307 \\
DOl: 10.1159/000329344
\end{tabular} & $\begin{array}{l}\text { Published online: } \\
\text { May } 28,2011\end{array}$ & $\begin{array}{l}\text { O } 2011 \text { S. Karger AG, Basel } \\
\text { ISSN } 1662-0631 \\
\text { www.karger.com/crg }\end{array}$ \\
\hline
\end{tabular}

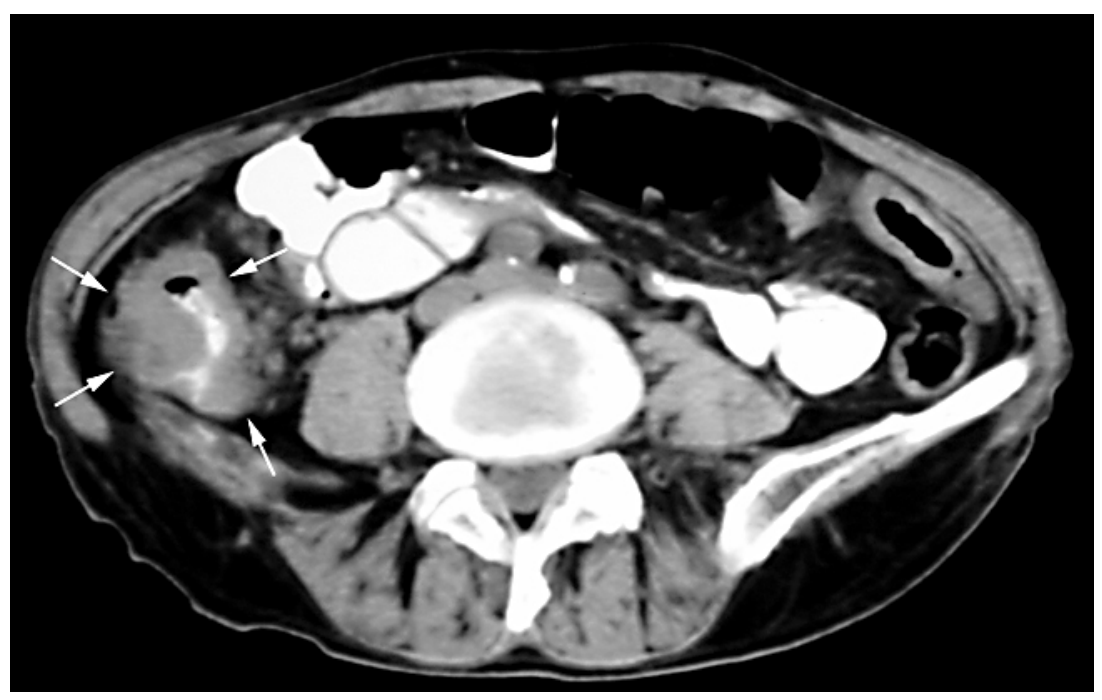

Fig. 2. Abdominal computed tomography scan demonstrating an irregular, large mass in the ascending colon (arrows) with partial luminal obstruction.

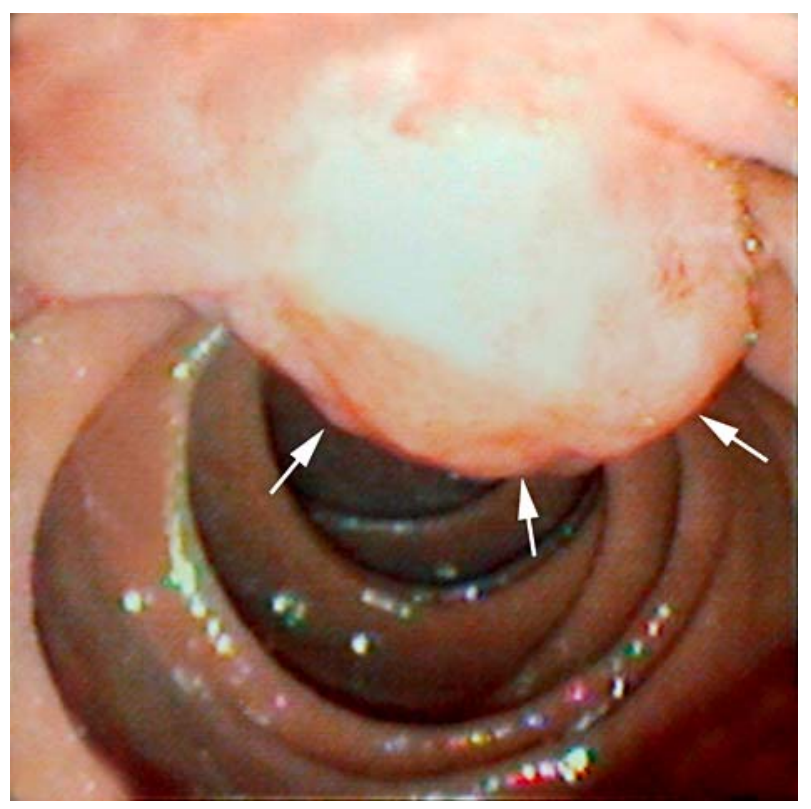

Fig. 3. Endoscopic appearance of the polypoid mass in the ampulla of Vater (arrows) which was proven to be a poorly differentiated ampullary adenocarcinoma. 


\begin{tabular}{r|l|l|l}
$\begin{array}{r}\text { Case Reports in } \\
\text { Gastroenterology }\end{array}$ & $\begin{array}{l}\text { Case Rep Gastroenterol 2011;5:301-307 } \\
\text { Dol: 10.1159/000329344 }\end{array}$ & $\begin{array}{l}\text { Published online: } \\
\text { May 28, 2011 }\end{array}$ & $\begin{array}{l}\text { O 2011 S. Karger AG, Basel } \\
\text { ISSN 1662-0631 } \\
\text { www.karger.com/crg }\end{array}$ \\
\hline
\end{tabular}

\section{References}

1 Mecklin JP, Järvinen HJ, Virolainen M: The association between cholangiocarcinoma and hereditary nonpolyposis colorectal carcinoma. Cancer 1992;69:1112-1114.

2 Järvinen HJ, Nyberg M, Peltokallio P: Biliary involvement in familial adenomatosis coli. Dis Colon Rectum 1983;26:525-528.

3 Lees CD, Hermann RE: Familial polyposis coli associated with bile duct cancer. Am J Surg 1981;141:378-380.

4 Bertoni G, Sassatelli R, Nigrisoli E, Pennazio M, Tansini P, Arrigoni A, Ponz de Leon M, Rossini FP, Bedogni G: High prevalence of adenomas and microadenomas of the duodenal papilla and periampullary region in patients with familial adenomatous polyposis. Eur J Gastroenterol Hepatol 1996;8:1201-1206.

$>5$ Kamisawa T, Egawa N, Tsuruta K, Okamoto A, Horiguchi S, Funata N: An investigation of primary malignancies associated with ampullary carcinoma. Hepatogastroenterology 2005;52:22-24.

-6 Su Y, Ahsan H, Neugut AI: The association between biliary tract cancers and cancers of other sites. Am J Gastroenterol 1999;94:2256-2262.

7 Das A, Neugut AI, Cooper GS, Chak A: Association of ampullary and colorectal malignancies. Cancer 2004;100:524-530.

8 Hatch EE, Curtis RE, Boice JD Jr, Fraumeni JF Jr: Malignant neoplasms associated with cancer of the ampulla of Vater. Br J Cancer 1992;66:1204.

-9 Moran A, Collins S, Evans DG, Davies R: Risk of subsequent primary cancers in patients with carcinoma of the ampulla of Vater. Br J Cancer 1997;76:1232-1233.

10 Ahmed F, Goodman MT, Kosary C, Ruiz B, Wu XC, Chen VW, Correa CN: Excess risk of subsequent primary cancers among colorectal carcinoma survivors, 1975-2001. Cancer 2006;107(5 suppl):1162-1171.

11 Evans HS, Lewis CM, Robinson D, Bell CM, Møller H, Hodgson SV: Incidence of multiple primary cancers in a cohort of women diagnosed with breast cancer in southeast England. Br J Cancer 2001;84:435-440.

12 Hemminki K, Li X, Dong C: Second primary cancers after sporadic and familial colorectal cancer. Cancer Epidemiol Biomarkers Prev 2001;10:793-798.

13 Baczako K, Büchler M, Beger HG, Kirkpatrick CJ, Haferkamp O: Morphogenesis and possible precursor lesions of invasive carcinoma of the papilla of Vater: epithelial dysplasia and adenoma. Hum Pathol 1985;16: 305-310.

14 Wittekind C, Tannapfel A: Adenoma of the papilla and ampulla - premalignant lesions? Langenbecks Arch Surg 2001;386:172-175.

15 Kaiser A, Jurowich C, Schönekäs H, Gebhardt C, Wünsch PH: The adenoma-carcinoma sequence applies to epithelial tumours of the papilla of Vater. Z Gastroenterol 2002;40:913-920.

- 16 Esposito I, Friess H, Büchler MW: Carcinogenesis of cancer of the papilla and ampulla: pathophysiological facts and molecular biological mechanisms. Langenbecks Arch Surg 2001;386:163-171.

17 Fischer HP, Zhou H: Pathogenesis of carcinoma of the papilla of Vater. J Hepatobiliary Pancreat Surg 2004;11:301-309. 